\title{
Line profile characteristics of solar explosive event bursts
}

\author{
Z. Ning, D. E. Innes, and S. K. Solanki \\ Max-Planck-Institut für Aeronomie, Max-Planck-Str. 2, 37191 Katlenburg-Lindau, Germany \\ Received 13 October 2003 / Accepted 3 February 2004

\begin{abstract}
The spatial structure and temporal evolution of explosive events are explored using spectral observations of the Si IV $1393 \AA$ Aine obtained with the Solar Ultraviolet Measurements of Emitted Radiation spectrograph (SUMER). Three areas of quiet Sun near disk center, $8^{\prime \prime} \times 120^{\prime \prime}$ each, were rastered with a cadence of less than $1 \mathrm{~min}$ for a period of over half an hour. Events were identified by a non-Gaussian excess in the blue and/or red wings of the line. We found 21 sites where events re-occur. The sites are clustered near regions of evolving network fields seen in low resolution Michelson Doppler Imager (MDI) magnetic field images. Events tend to expand and shrink across the surface with a speed $\sim 25 \mathrm{~km} \mathrm{~s}^{-1}$. Individual events were also seen to move at this speed across the solar surface. Consecutive events often have different line profile characteristics and sizes, suggesting changes in the structure of the accelerated plasma from event to event. In the majority of events, blue and red wing brightenings do not produce a simultaneous line intensity increase. Also, the red and blue wing emission is mostly cospatial implying either non-directed flow or jets orientated along the line-of-sight (i.e. out from and towards the solar surface). The explosive events within a burst are in some cases separated by 3-5 min, suggesting that oscillations, which are known to have such periods, may play a role in triggering the individual events of a burst.
\end{abstract}

Key words. Sun: transition region - Sun: oxillations - Sun: UV radiation - line: profiles

\section{Introduction}

Explosive events are one of the best studied dynamic phenomena of the transition region. Based on observations from several High Resolution Telescope and Spectrograph (HRTS) flights, Dere et al. (1989) and Dere (1994) described the basic properties of explosive events and their relationship to the photospheric magnetic field. Explosive events are detected most frequently in transition region lines formed typically around $10^{5} \mathrm{~K}$, and rarely in lines formed below $3 \times 10^{4} \mathrm{~K}$ or above $3 \times 10^{5} \mathrm{~K}$. They are characterized by broad line profiles with high velocity components of typically $\sim 100 \mathrm{~km} \mathrm{~s}^{-1}$, a spatial size $\sim 2^{\prime \prime}(1500 \mathrm{~km})$, and an average lifetime $\sim 1 \mathrm{~min}$. They have a high birthrate of $10^{-20} \mathrm{~cm}^{-2} \mathrm{~s}^{-1}$ in the quiet Sun and coronal holes. From observations with the Solar Ultraviolet Measurement of Emitted Radiation (SUMER) spectrograph on board the Solar and Heliospheric Observatory (SOHO), Innes et al. (1997b) deduced that explosive events are the signatures of the outflow jets associated with magnetic reconnection. This condition is strengthened by joint SUMER and Big Bear Solar Observatory (BBSO) observations. From these Chae et al. (1998) found that explosive events tend to occur above regions with weak and mixed polarity magnetic flux in the photosphere. The photospheric evolution occurs on a time scale of several hours whereas the explosive events last for about 1-4 min.

Send offprint requests to: D. E. Innes,

e-mail: innes@linmpi.mpg.de
They therefore suggest that explosive events represent fast magnetic reconnection in the transition region, which is initiated by slow magnetic evolution in the photosphere underneath.This agrees with the high velocity bi-directional structure of explosive events (Innes et al. 1997b) and the explosive event bursts seen by Innes et al. (1997a) and Chae et al. (1998).

Until now, studies of explosive event bursts have only been made when the spectrometer slit was pointing at one position of the event, so that the overall event profiles and their structure could not be seen. Thus it was unclear whether explosive events exhibit a bursty behaviour because they switch on and off or because they move in and out of the spectrograph slit. In this paper we describe the line profile behaviour across whole events in several explosive event bursts.

\section{Observations and data reduction}

The observations were made with SUMER (Wilhelm et al. 1995, 1997; Lemaire et al. 1997) on 21 June 1996 during solar minimum near the disk center. Figure 1 shows the region rastered superimposed on the low resolution Michelson Doppler Imager (MDI; Scherrer et al. 1995) images that are closest in time to the beginning and end of the observing sequence. As can be seen, the region covered several network lanes that showed signs of photospheric flux evolution.

SUMER obtained stigmatic images along its $1^{\prime \prime} \times 120^{\prime \prime}$ slit with a resolution of $1^{\prime \prime}$ and $9 \mathrm{~km} \mathrm{~s}^{-1}$ in SiIV $1393 \AA$. 


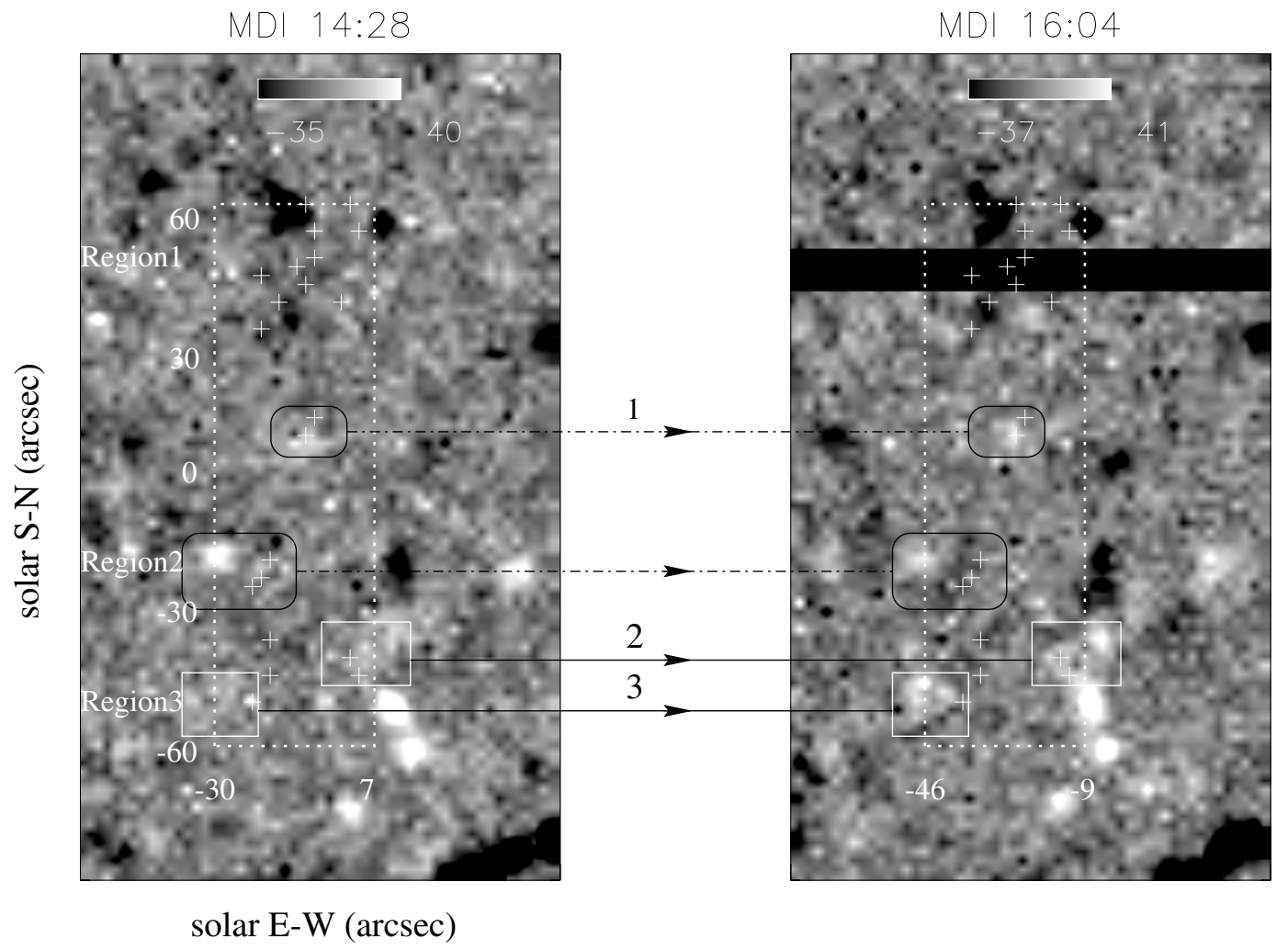

Fig. 1. Photospheric magnetic fields from MDI observations at 14:28 and 16:04 on June 21, 1996. The black bar at 16:04 is due to missing MDI data. The box outlined with dots indicates the area rastered by SUMER and the crosses mark the locations of explosive events. Two zones with increasing magnetic flux (emergence) are marked with white squares. A reduction of the magnetic flux (cancellation) is seen in the two regions inside the black boxes.

Our observations were made for three different periods of about half an hour each. In half an hour, the Sun rotates about 5", so that the area of the Sun rastered at the beginning of the period had virtually rotated out of the field-of-view by the end of the half hour. This rotation is clear in the explosive event site motion, as we shall see later.

In each period, small raster scans, either $5.63^{\prime \prime}$ or $7.88^{\prime \prime}$ wide in the $\mathrm{E}-\mathrm{W}$ direction, were made. The rasters were made with either 6 or 8 steps of size 1.125". An exposure time of either $5 \mathrm{~s}$ or $10 \mathrm{~s}$ was used depending on the observing sequence. In order to improve the time resolution, the area was first observed at the odd positions and then at the even positions. Thus the area was actually scanned in half the number of steps with gaps at every second position. The missing positions were then observed in the next scan. The observing scheme is illustrated in Fig. 2. A careful look at this figure shows there are significant profile changes in both the spatial and temporal direction between consecutive spectra. We find, however, that after looking at many such rasters that the changes in the temporal direction are smoother than in the spatial direction. Therefore in the following analysis, we have filled the missing raster positions with the average of the spectra taken just before and after at the same position. Some of the data from this series of observations have been presented in Innes et al. (1997b).

Si IV $1393 \AA$ is a strong, isolated transition region line and has been used in several studies of explosive events with SUMER (Innes et al. 1997b; Chae et al. 1998, 2000).

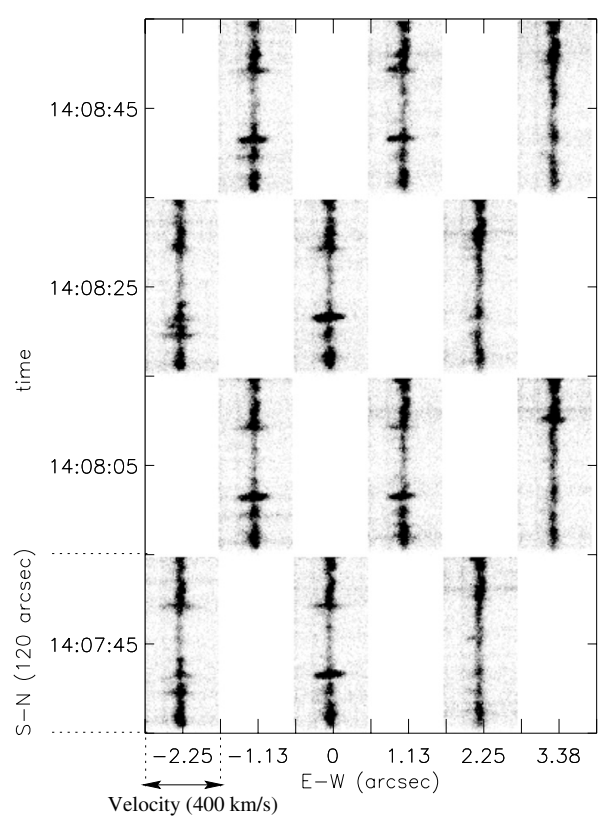

Fig. 2. Temporal and spatial structure of the Si IV $1393 \AA$ A line during two raster scans. Each raster scan consists of 6 steps in the east-west direction, with a distance of $1.13^{\prime \prime}$ between steps. The exposure time of each image is $5 \mathrm{~s}$, and each raster takes $40 \mathrm{~s}$. First the odd positions were observed (2.25" between steps) and then the even positions. Each unit is $120^{\prime \prime}$ from bottom to top and runs form $-200 \mathrm{~km} \mathrm{~s}^{-1}$ (blue shift) on the the left to $+200 \mathrm{~km} \mathrm{~s}^{-1}$ on the right. 


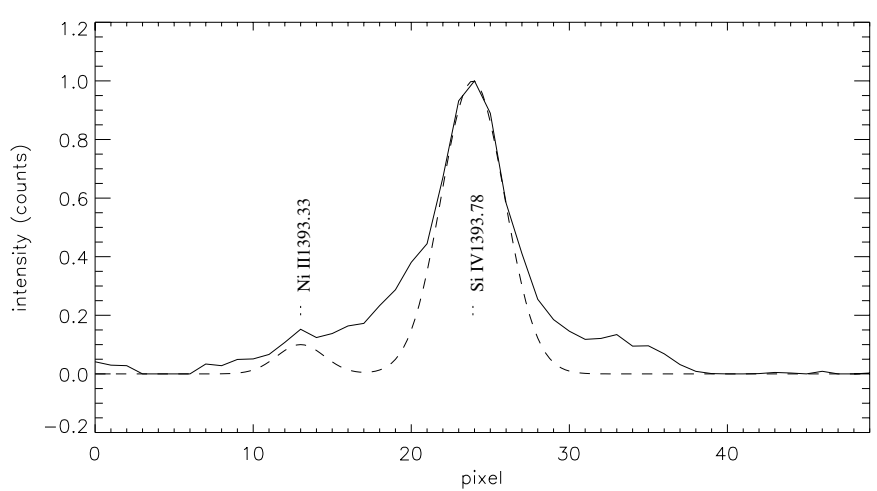

Fig. 3. Average quiet Sun emission (dashed line) around the SiIV $393.78 \AA$ line and an explosive event (solid line). The Ni II $1393.33 \AA$ line blends with the blue wing about 10 to 11 pixels away from the Si IV line center. Ni II has a peak intensity about one tenth of the Si IV peak, as can be judged from the profiles with no explosive events.

To obtain the Si IV line emission, background emission from the continuum and the Ni II line at $1393.33 \AA$ have to be subtracted (see Fig. 3). The continuum level is computed by interpolating the average counts in two windows on either side of the line, between pixels 5-8 and 43-48. The strength of the Ni II $1393.33 \AA$ line is more difficult to estimate especially when there is a broad Si IV blue wing. Since Ni II is formed at too low a temperature to exhibit explosive events, it can often be removed by fitting a Gaussian of width $20 \mathrm{~km} \mathrm{~s}^{-1}$ at the wavelength position of the line. When the Si IV wing reaches out to the Ni II line, line fitting is inappropriate. In such cases, we assumed that the Ni II line center intensity is a fixed, 10\%, fraction of the Si IV line center value unless this was larger than the observed intensity at the Ni II wavelength. In which case the $\mathrm{Ni}$ II line center intensity was taken to be equal to the observed value. The Si IV blue wing intensity may be underestimated in these very rare cases.

The Si IV line profile can be thought of as a low velocity core component that reflects density and/or ionization changes in the plasma, and higher velocity red and blue wings that reflect line-of-sight motion. The profiles are analysed by fitting a line core of standard width, $20 \mathrm{~km} \mathrm{~s}^{-1}$, to the observed profile to obtain the line shift and maximum intensity. The excess counts in the red and blue non-Gaussian wings are added up to determine the strength of the high velocity component. This same method for detecting explosive events was used by Innes (2001).

\section{Examples and results}

\subsection{Repetition of explosive events}

First each profile was analysed as described in Sect. 2 to obtain the 2D time evolution of the intensity and line shift of the Si IV core, and the intensity in the non-Gaussian blue and red wings. Figure 4 shows examples of the $2 \mathrm{D}$ wing and core intensity structure at two different times (hereinafter P1 and P2). These two pictures suggest that wing and core brightenings are neither co-spatial nor co-temporal. In the event between solid
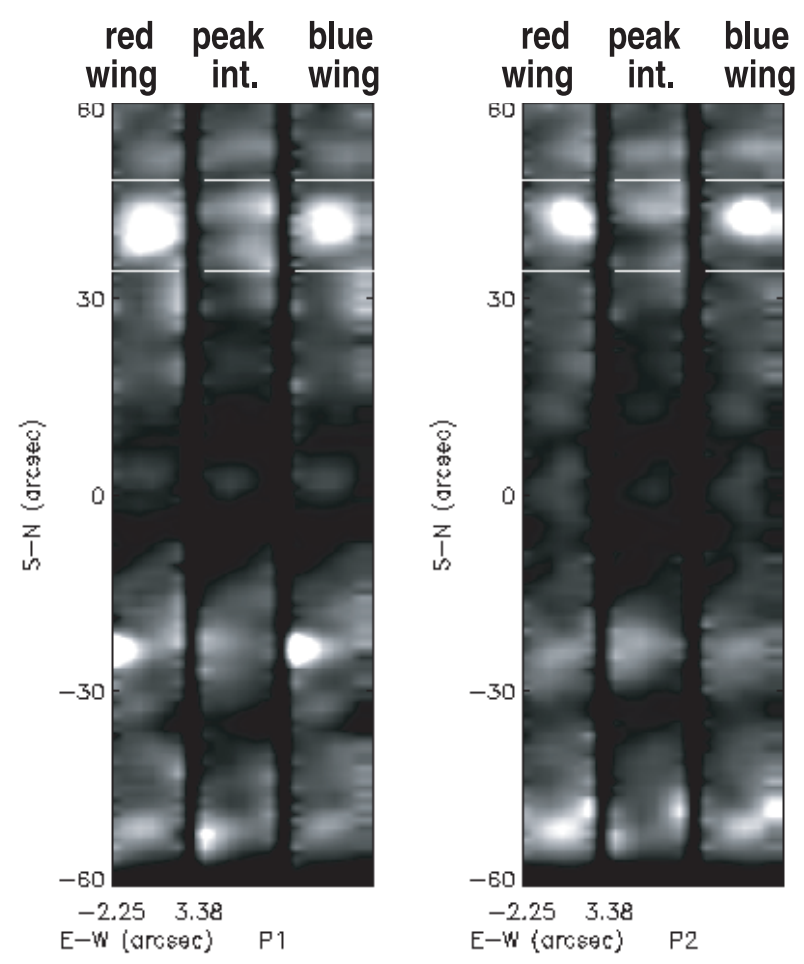

Fig. 4. Snapshot of blue and red wing and core intensity during two raster scans taken around 16:54:24 UT (left) and around 16:58:29 UT (right). The white boxes outline events P1 and P2.

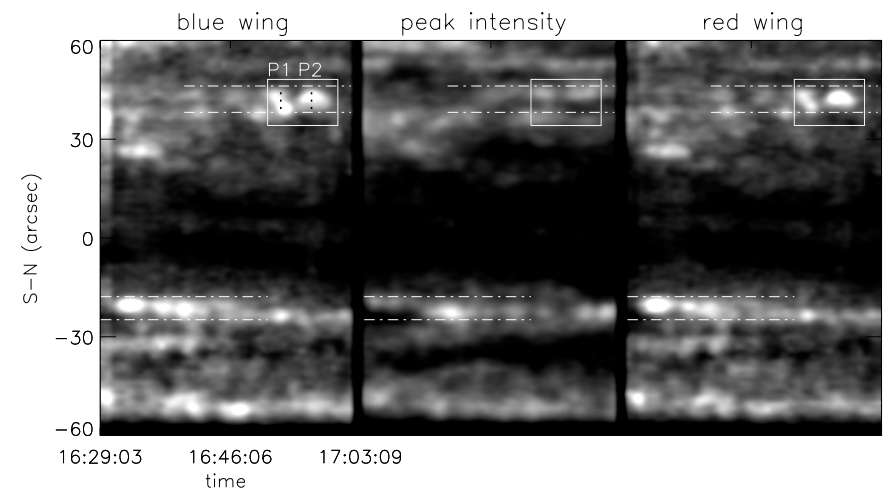

Fig. 5. Time variation of blue wing, peak and red wing intensity during the last half hour observation period, after integration in the E-W direction. The E-W behaviour of the two explosive event sites bounded by white dash-dot lines is shown in Figs. 6a and 6b. The snapshots shown in Fig. 4 were taken at the times of P1 and P2.

lines at $\mathrm{P} 1$, the wing brightening seems to coincide with a dark area between small patches of brighter core emission. Innes (2001) suggested that the core intensity brightened one or two minutes after the wings. With this dataset we see that it may brighten not only after but also to the side of the wing.

In order to obtain an overview of the time evolution along the slit the data were first summed in the E-W direction. Figure 5 shows the blue wing, peak and red wing intensity as a function of time for the last of the half hour periods. The more active, brighter emission in the north is coming from the 

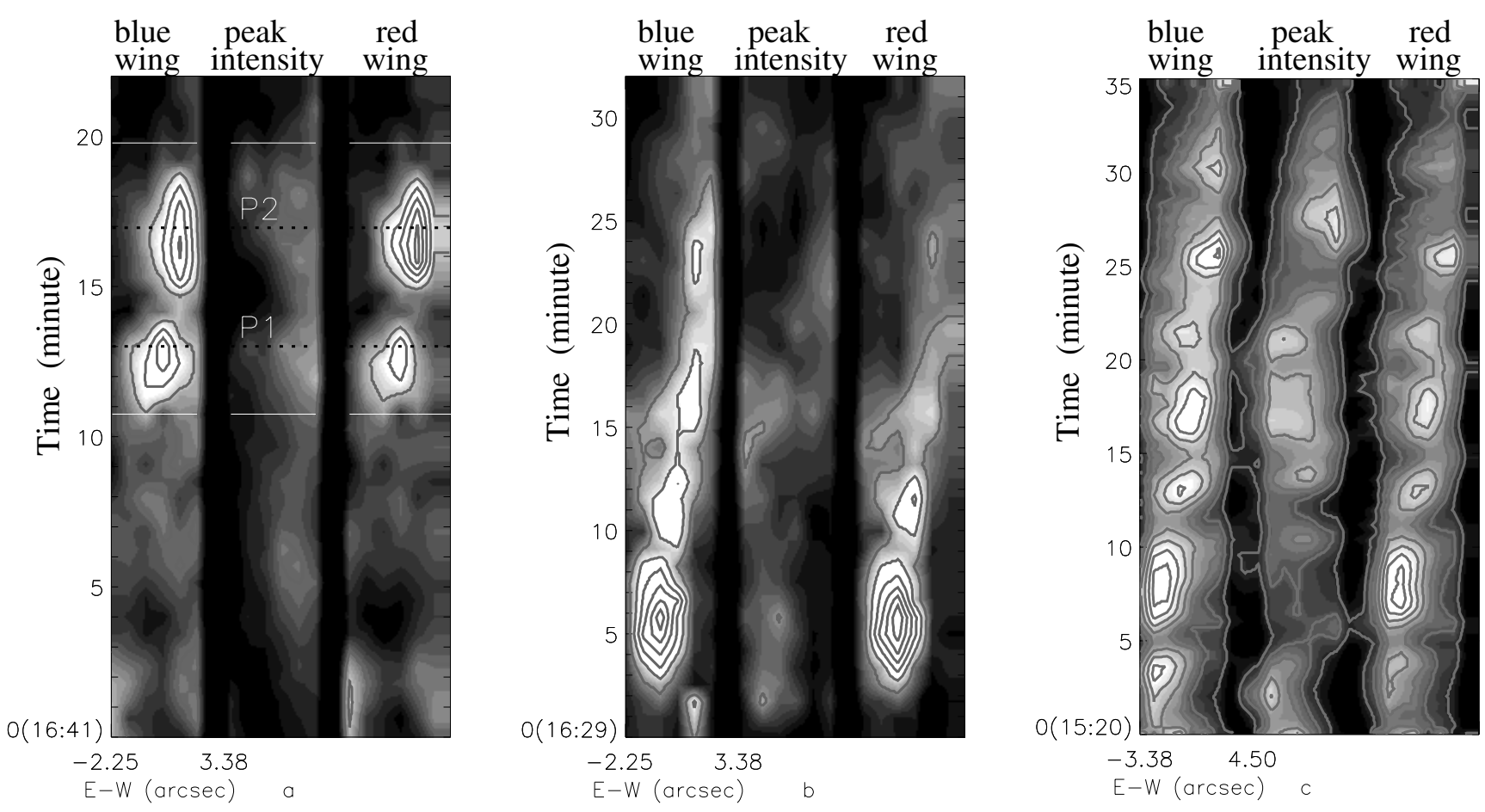

Fig. 6. Examples of re-occuring explosive events. The east to west movement is due to solar rotation (1" $/ 6$ min). The events in a) are $P 1$ and $P 2$ (Fig. 4). The four events in b) are the southern string of bright events between dot dashed lines in Fig. 5. The site in c) with 7 repetitions, is the one with the maximum number of repetitions in our dataset.

region with many events below the strong negative field region at the north in Fig. 1. The southern part of the slit crosses regions 2 and 3 in Fig. 1. In between, where the magnetic field is weaker, corresponding to a network cell center, the emission is much lower. Again, explosive events do not co-incide with core brightenings.

The wing and core brightenings seem to occur in bursts lasting about 2-4 min. This can be seen in Fig. 5 in the boxed region to the north and between the dot-dashed lines near $\mathrm{S}-\mathrm{N}=-30^{\prime \prime}$. In this representation, the evolution of N-S active sites can be seen but the E-W evolution is lost. The E-W structure of the two bright events in Fig. 5 are displayed in Figs. $6 \mathrm{a}$ and $6 \mathrm{~b}$, by integrating over the $\mathrm{N}-\mathrm{S}$ direction between the dotdashed lines. The northern one flares up twice and the other one three times. Figure $6 \mathrm{c}$ shows a multi-recurrence burst in the second period. The events move from east to west on the solar disk due to the Sun's rotation.

The whole dataset was analysed by looking at the time evolution of each $\mathrm{N}-\mathrm{S}$ pixel, after summing over the E-W direction. In this way active N-S sites could be identified (see Fig. 5). Figure 7 shows the time evolution of the peak, the blue and the red wing intensity, summed in the E-W directions, for 21 event sites identified during the three periods. Explosive events are those with large increases in the blue (dotted line) and/or red (dash-dotted line) wing intensities. The total flux of the central component is the peak intensity times a constant of $\sqrt{\pi} \sigma \sim 3.2$, where $\sigma=1.8$ is the assumed Doppler width in pixels of the Si IV central component. Only those marked by the tick marks on the bars above or below are from the same location on the Sun. The other explosive events are mostly those at sites either on the west edge at the beginning of the half hour or the east edge at the end of the half hour, so that the site is only in the field-of-view for a short period. In most events the red and blue wings increase/decrease simultaneously, but there is no regular correlation with the peak intensity. For example, site 3 in Fig. 7 has three explosive events. During the first two events the central component hardly brightens at all, but the next time all three components brighten almost simultaneously. This example is exceptional in that the core brightening slightly precedes the wing. In the majority of events with almost simultaneous wing and core brightening the core lags behind the wing (e.g. the last event at site 9, or the first event at site 11).

The site with the most repetitions is site 6 . At this position seven bursts are seen. In the half hour observing sequence the site crosses from the western to the eastern edge of the fieldof-view (Fig. 6c). The Si IV peak intensity also shows repeated brightening, which is poorly correlated with the wing brightenings. The median time interval between repetitions is less than $5 \mathrm{~min}$, which is the same order as that given in Chae et al. (1998). In a few cases much longer repetition times are also observed (e.g. at sites 11 and 19).

\subsection{Explosive event spatial structure}

Each event in this dataset is unique but every one either moves across the surface and/or expands in the N-S or E-W direction. We can use the two examples, P1 and P2, to measure typical speeds. Figure 8 shows these two events in detail. In the first event, $\mathrm{P} 1$, the event center moves about $2^{\prime \prime}$ southward with a velocity of $\sim 30 \mathrm{~km} \mathrm{~s}^{-1}$. There is no measureable E-W movement. The second event expands and contracts northward with a similar speed, $\sim 25 \mathrm{~km} \mathrm{~s}^{-1}$, and eastward at $\sim 12 \mathrm{~km} \mathrm{~s}^{-1}$. 

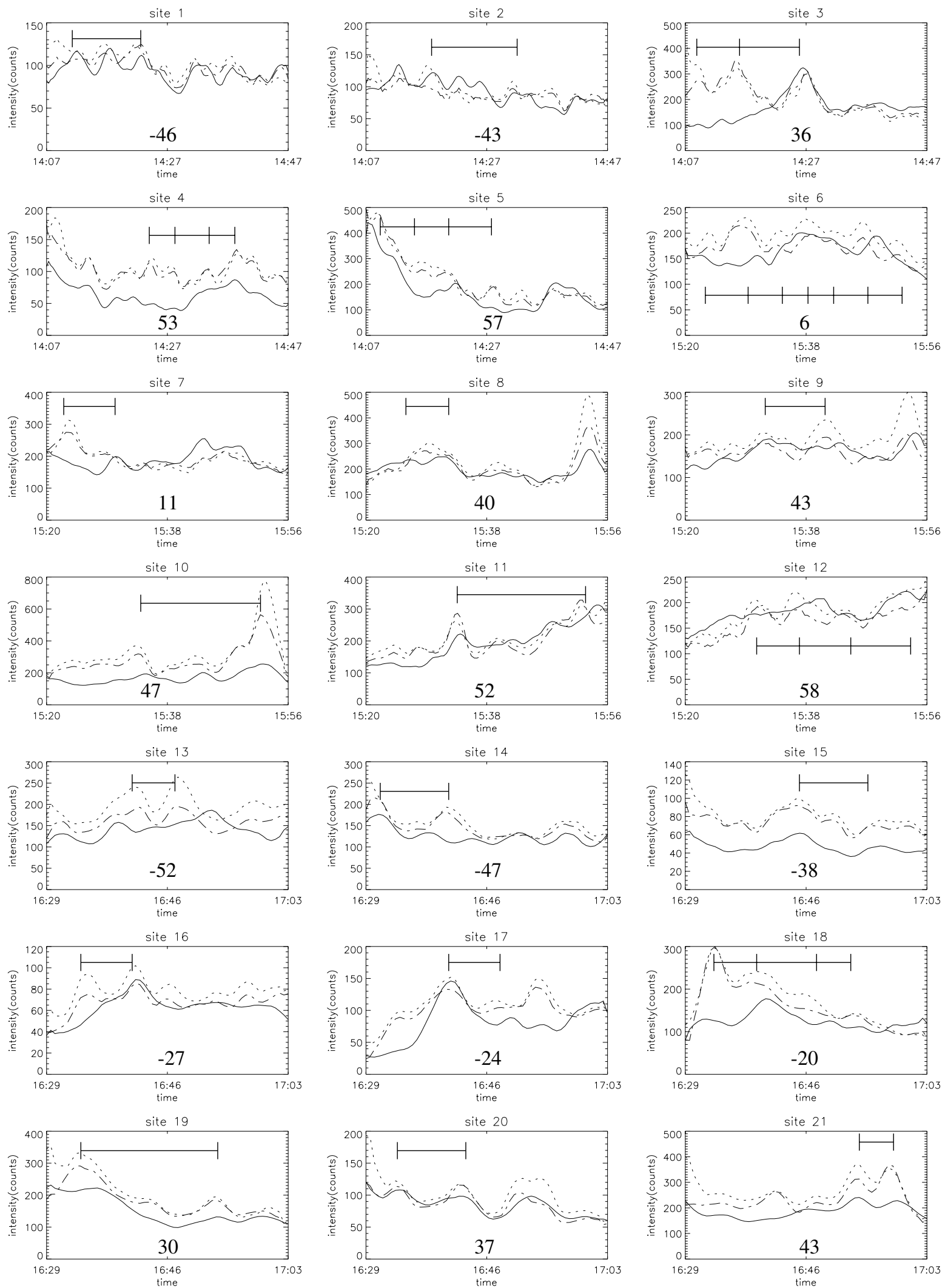

Fig. 7. Time variation of peak (solid), blue wing (dotted) and red wing (dash-dotted) intensity integrations at various N-S positions for 21 sites with repetitive explosive events from three different periods. The N-S position is marked on each frame. The peaks produced by repeating events are marked. Some are partly washed out by the E-W integration. There are also unmarked peaks due to events on the east or west edges of the raster. Sites 21, 18 and 6 correspond to the three sites in Figs. 6a-c, respectively. 

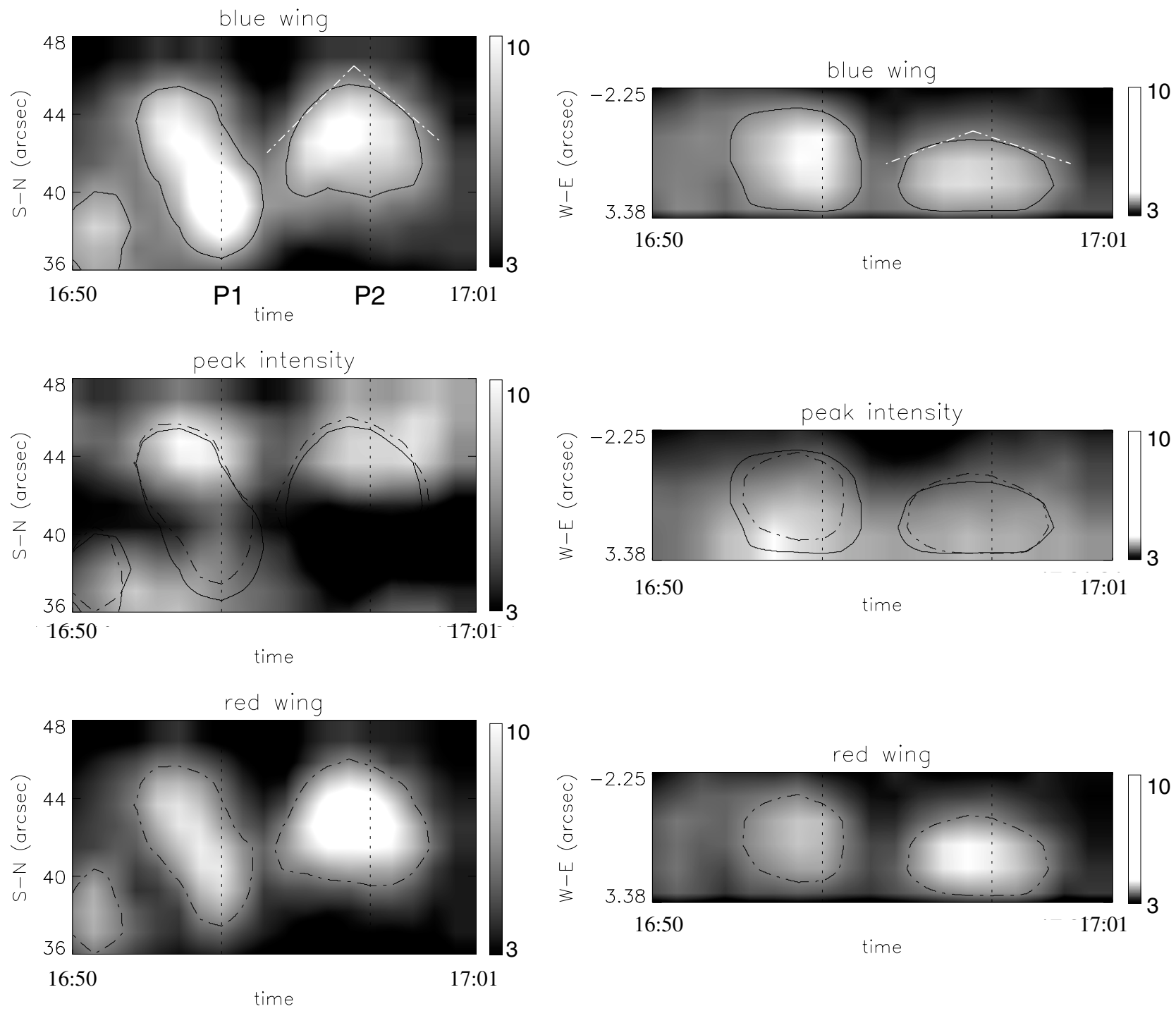

Fig. 8. The expansion and contraction across the solar surface in the S-N and E-W directions of explosive events P1 and P2. The contours are at 7 counts $\operatorname{arcsec}^{-2}$ per exposure time (15 s), solid lines for the blue wing and dashed lines for the red wing. These contours are overplotted on the line core intensity images. The expansion and contraction speeds of $\sim 25 \mathrm{~km} \mathrm{~s}^{-1}$ to the north and south and $\sim 12 \mathrm{~km} \mathrm{~s}^{-1}$ to the east and west are computed from the slopes (white dot-dashed lines) after line-fitting the contours.

The expansion and contraction speeds have been measured by fitting a straight line (examples are the white dot-dashed lines) to the contours (black solid lines) in Fig. 8. The event speed across the solar surface is roughly the sound speed of plasma at the temperature of the transition region, $\sim 6 \times 10^{4} \mathrm{~K}$. It is about a factor three less than the maximum line-of-sight velocity deduced from the line wing. This is understandable if the flow is directed predominantly along the line-of-sight or if the accelerated plasma is pushing against dense chromospheric plasma (cf. models of Innes \& Tóth 1999).

The 2D time evolution of several of the events is shown in Fig. 9. In this representation the 3D data has been projected onto a plane. The gray-scale indicates the maximum, not the integrated, intensity along a projected path. Thus it is good for showing the overlap of the bright components but not the fainter structure. The first column shows the blue wing intensity with the red wing contours superimposed, the second shows the red wing with the red wing contours superimposed and the third the core intensity with the same red wing contours. Probably the most striking feature is the low correlation between core and wing brightening and the high correlation between red and blue wing brightening.

There are a couple of more subtle points. The first is the number of events with spatial offset between the red and blue wing brightening. This feature was interpreted by Innes et al. (1997b) as evidence of reconnection jets. There are four events with obvious red-blue offsets, enclosed in black dashed line boxes in the first column of Fig. 9. In two of these, the blue wing is significantly more extended than the red suggesting greater expansion into the corona than towards the solar surface. The majority of events have co-spatial red and blue wing brightening. It may be argued that at disk center, where these observations were taken, the red and blue components are mostly co-spatial because the jets are predominantly perpendicular to the solar surface. We note that the largest red-blue spatial offset in Innes et al. (1997b) was seen from an event at 


\section{blue wing}
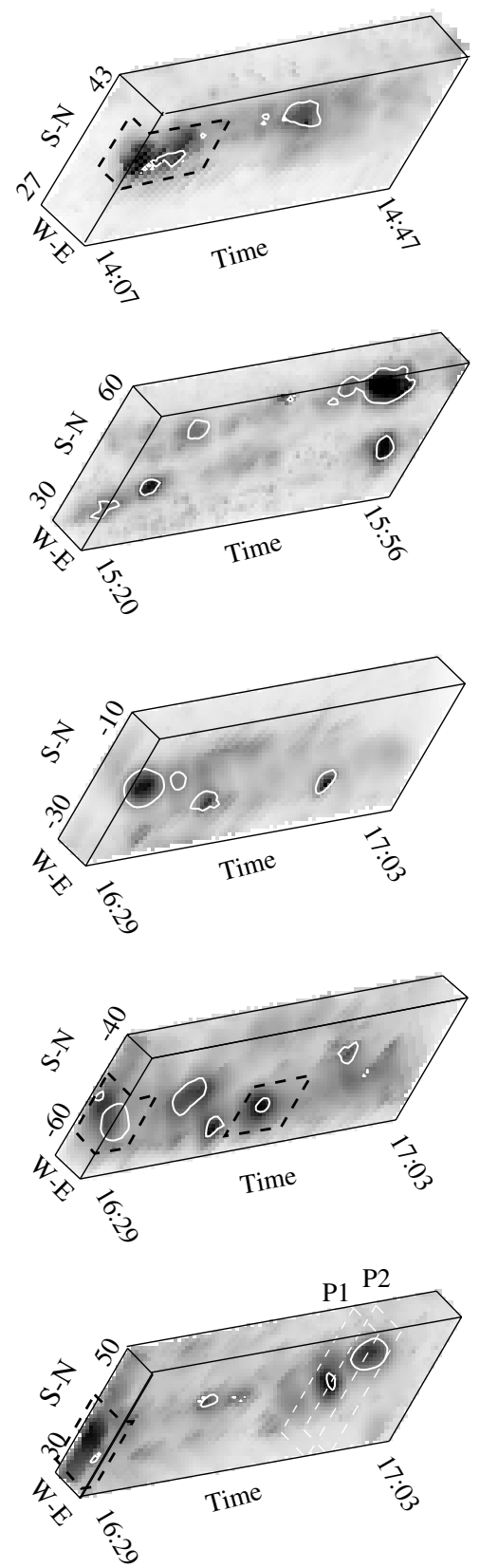

red wing
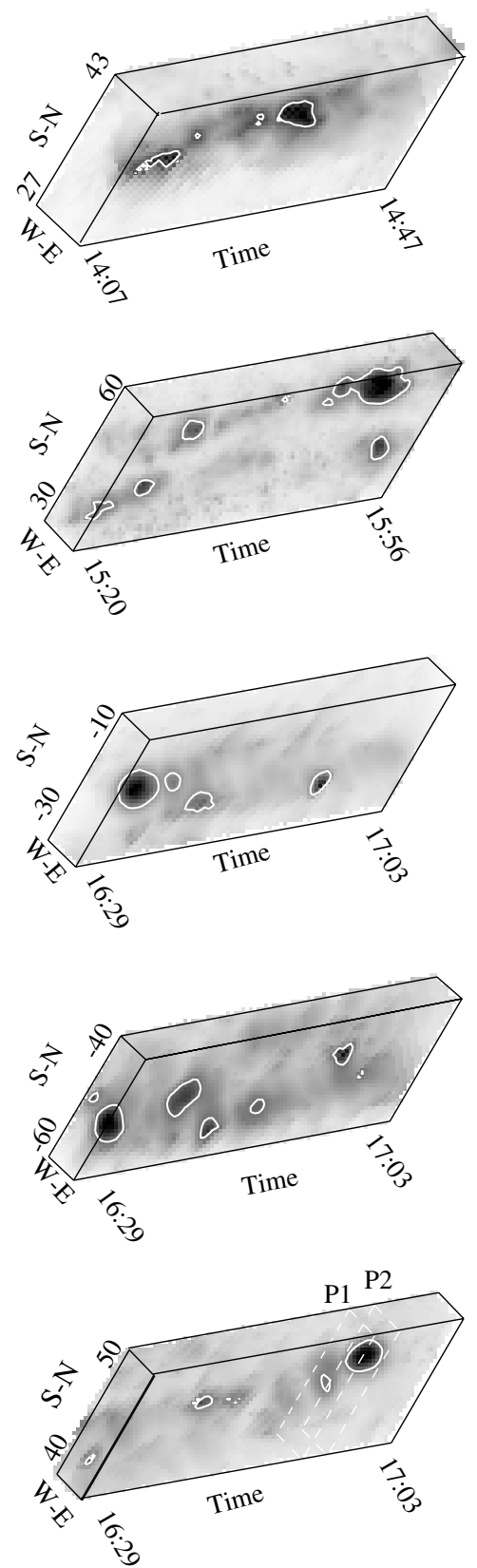

\section{peak intensity}
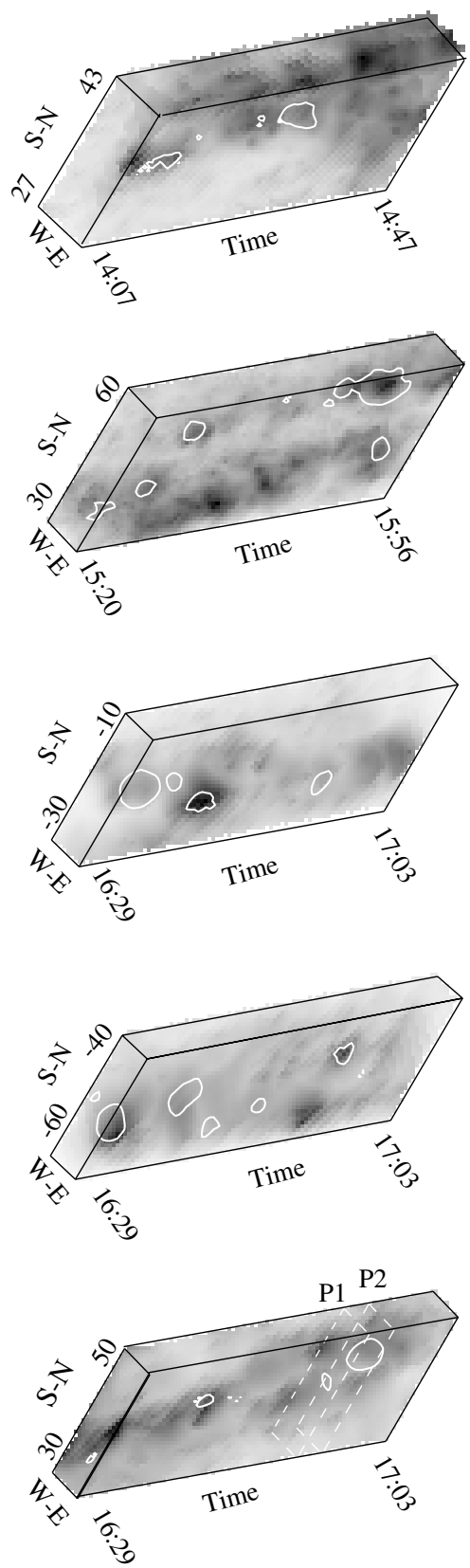

Fig. 9. The 2D time evolution of 5 groups of events. Each row shows the blue wing intensity (Col. 1), the red wing intensity (Col. 2) and peak intensity (Col. 3) with red wing intensity contours superimposed on each image. The 3D data has been projected onto a plane, producing a translucent image of the data (like an X-ray). The brightest data points along the projected path are darkest. Time runs left to right. S-N is as indicated on the vertical and the full E-W raster makes up the short side. The location of P1 and P2 are indicated. The black dashed line boxes surround events with obvious red-blue wing spatial offset.

solar latitude $60^{\circ}$. Theoretically jets of all orientations are expected (Yokoyama \& Shibata 1996) and are seen in active regions (Shimojo et al. 1996). Further analysis of explosive event observations at large latitudes are required to clarify this point.

The second point relates to blinkers (Harrison 1997; Harrison et al. 1999). As can be seen in the last column of Fig. 9, the longer and larger brightenings (e.g. the one in the north of row 1 and in the south of row 2) do not have simultaneous wing brightening. This is consistent with observations of blinkers. Some small single bursts of core brightening do have associated wing brightening (e.g. the last event in row 2, the third event in row 3 ).

\subsection{The magnetic field}

The positions of the explosive event sites are shown in Fig. 1. In agreement with the results of Chae et al. (1998), we also find that most sites can be associated with either emerging or 
cancelling flux. The site with the most repetitions (site 6) is in the cancelling flux region 2 . There is no site on top of the strong unipolar field region in the north, although there is a site just at its edge. The region to the south with many explosive event sites is unfortunately missing from the later MDI image, so that we cannot judge if new magnetic flux emerged there.

\section{Discussion}

The activity of the transition region has been well documented in a number of earlier publications. It is well-known that sites of evolving magnetic field are the sites of explosive events and transition region brightenings (Dere et al. 1991; Chae et al. 1998; Innes 2001). The study presented here has taken our knowledge a step further by analyzing the structure of the events to see whether event characteristics persist during the activity phase, and in particular how the events evolve spatially and temporally.

We have shown that bursts of explosive events exist and that between two events in a burst the profiles revert almost to their quiet Sun shape everywhere in the raster. The recurrence rate between events in a burst is often close to 3-5 min, similar to the period of chromospheric and transition region oscillations. This indicates that reconnection events within a burst could be triggered at a particular phase of a wave or oscillation. Compressive waves push the field lines together at certain phases, which may well induce reconnection if opposite polarity fields are present. Similarly, a torsional Alfvén wave periodically changes the angle between the field lines of the flux tube carrying the wave and a neighbouring field. Reconnection is most likely at phases when this angle is maximised.

We also find that event characteristics change from event to event within a site, suggesting re-configuration of the active site. In most events the red and blue wing emission is cospatial implying either non-directed flow or jets oriented along the line-of-sight. This in turn implies, since the observations were taken at disk center, that any jet-like flow is predominantly out from and towards the solar surface. Further study of these and other events is required to establish the flow geometry at different positions on the solar disk.

Acknowledgements. The SUMER project is financially supported by DLR, CNES, NASA and the ESA PRODEX programme (Swiss contribution). SUMER is part of SOHO, the Solar and Heliospheric Observatory, of ESA and NASA. Ning Zongjun would like to thank Prof. Dr. Wang Jinsong, Dr. Xia Lidong and Dr. Wang Tongjiang for their kind help.

\section{References}

Chae, J., Wang, H., Goode, P. R., Fludra, A., \& Schühle, U. 2000, ApJ, 528, L119

Chae, J., Wang, H., Lee, C., Goode, P. R., \& Schühle, U. 1998, ApJ, 497, L109

Dere, K. P. 1994, Adv. Space Res., 14, 13

Dere, K. P., Bartoe, J.-D. F., \& Brueckner, G. E. 1989, Sol. Phys., 123, 41

Dere, K. P., Bartoe, J.-D. F., Brueckner, G. E., Ewing, J., \& Lund, P. 1991, J. Geophys. Res., 96, 9399

Harrison, R. A. 1997, Sol. Phys., 175, 467

Harrison, R. A., Lang, J., Brooks, D. H., \& Innes, D. E. 1999, A\&A, 351,1115

Innes, D. E. 2001, A\&A, 378, 1067

Innes, D. E., Brekke, P., Germerott, D., \& Wilhelm, K. 1997a, Sol. Phys., 175, 341

Innes, D. E., Inhester, B., Axford, W. I., \& Wilhelm, K. 1997b, Nature, 386, 811

Innes, D. E., \& Tóth, G. 1999, Sol. Phys., 185, 127

Lemaire, P., Wilhelm, K., Curdt, W., et al. 1997, Sol. Phys., 170, 105

Scherrer, P. H., Bogart, R. S., Bush, R. I., et al. 1995, Sol. Phys., 162, 129

Shimojo, M., Hashimoto, S., Shibata, K., et al. 1996, PASJ, 48, 123

Wilhelm, K., Curdt, W., Marsch, E., et al. 1995, Sol. Phys., 162, 189

Wilhelm, K., Lemaire, P., Curdt, W., et al. 1997, Sol. Phys., 170, 75

Yokoyama, T., \& Shibata, K. 1996, PASJ, 48, 353 